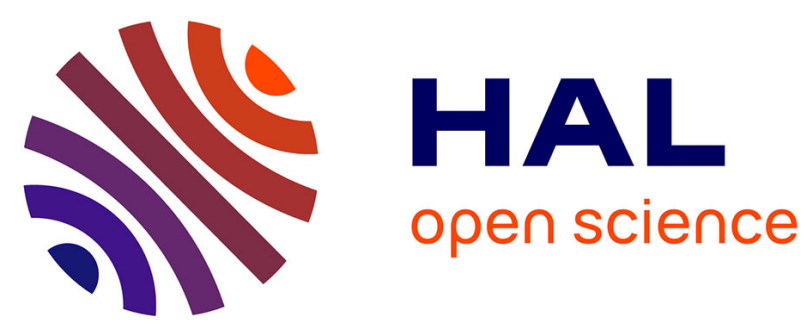

\title{
Multicellular Zero Forcing Precoding Performance in Rayleigh and Shadow Fading
}

Dorra Ben Cheikh, Jean-Marc Kélif, Marceau Coupechoux, Philippe

Godlewski

\section{- To cite this version:}

Dorra Ben Cheikh, Jean-Marc Kélif, Marceau Coupechoux, Philippe Godlewski. Multicellular Zero Forcing Precoding Performance in Rayleigh and Shadow Fading. IEEE Vehicular Technology Conference, VTC Spring, May 2011, Budapest, Hungary. pp.1-5. hal-00717290

\section{HAL Id: hal-00717290 \\ https://hal-imt.archives-ouvertes.fr/hal-00717290}

Submitted on 12 Jul 2012

HAL is a multi-disciplinary open access archive for the deposit and dissemination of scientific research documents, whether they are published or not. The documents may come from teaching and research institutions in France or abroad, or from public or private research centers.
L'archive ouverte pluridisciplinaire HAL, est destinée au dépôt et à la diffusion de documents scientifiques de niveau recherche, publiés ou non, émanant des établissements d'enseignement et de recherche français ou étrangers, des laboratoires publics ou privés. 


\title{
Multicellular Zero Forcing Precoding Performance in Rayleigh and Shadow Fading
}

\author{
Dorra Ben Cheikh ${ }^{\dagger \star}$, Jean-Marc Kelif^${ }^{\star}$, Marceau Coupechoux ${ }^{\dagger}$ and Philippe Godlewski ${ }^{\dagger}$ \\ * Orange Labs \\ Issy-les-moulineaux, France \\ Email: \{dorra.bencheikh, jeanmarc.kelif\}@ orange-ftgroup.com \\ ${ }^{\dagger}$ TELECOM Paris Tech \& CNRS LTCI \\ Paris, France \\ Email: $\{$ bencheikh, coupecho, godlewski\}@enst.fr
}

\begin{abstract}
In this paper we propose an analytical evaluation of the performance of the zero forcing precoding technique in terms of outage probability in a multicellular multiuser context. The channel model includes path loss shadowing and Rayleigh flat fading. Two cases are examined. The first one considers a constant lognormal shadowing. In this case, a closed form expression of the outage probability is derived. In the second case we consider a randomly variable lognormal shadowing and we propose an easily computable expression of the outage probability. Simulation results show the degradation of performance induced by the shadowing.

Index Terms-Zero forcing, multicellular, multiuser, outage probability, lognormal shadowing.
\end{abstract}

\section{INTRODUCTION}

MIMO (Multiple inputs multiple outputs) systems have been promoted as a technique promising attractive enhancement in the quality of service [13] and capacity [8]. In a multiuser context, the study of this technology is even more interesting since it permits to benefit from multiuser diversity and to achieve higher capacity. In this context, many multiuser transmission techniques have been proposed. The Dirty Paper Coding (DPC) [1] was proposed as an optimal transmission technique allowing a partial pre-cancellation of the interference at the transmitter without any increase in the transmit power. The DPC was proven to achieve the capacity region of MIMO broadcast channels [10]. However, the implementation complexity induced by the DPC makes it excluded from usage in practice. As an alternative, linear precoding as Zero Forcing ( $\mathrm{ZF}$ ) and Minimum Mean Squared Error (MMSE) have been proposed. These techniques are suboptimal but present the advantage of a low implementation complexity. The ZF precoding allows a total cancellation of the multiuser interference at the price of an increase in the transmit power depending on the channel conditioning. The MMSE precoder is a technique achieving a trade-off between the interference cancellation and the transmit power increase. These two techniques have been studied in [7] in terms of symbol error rate. Analytical distributions of the signal-tointerference-plus-noise ratio (SINR) of a ZF precoded system was proposed for a broadcast channel in a single cell system for a Rayleigh channel without considering shadowing. In [9], the performance of the $\mathrm{ZF}$ precoding was investigated in the case of imperfect channel estimation in terms of bit error rate for a broadcast single cell MIMO system. In [6], an asymptotic analysis of linear precoding techniques ( $\mathrm{ZF}$ and MMSE) was conducted for small cells multiuser multicellular systems where the inter-cell interference affects enormously the system performance due to the small distance between cells. The authors showed a dramatic decrease in the achievable sum-rate due to multicell interference. In [11] and [12], it was shown that for a MIMO broadcast channel and using an adequate user selection algorithm, the $\mathrm{ZF}$ precoding approaches the performance of the DPC for a large number of users in the cell.

Regarding its implementation simplicity and its potential of optimality, we will focus on the ZF precoding technique. In the literature, the ZF performance has been studied in a single cell context without considering multicell interference and for a Rayleigh channel except in [6] where the multicellular case has been studied in terms of sum-rate capacity for small-cell systems. In this work, we propose an analytical study of the performance of the $\mathrm{ZF}$ precoding in terms of outage probability in a multiuser multicellular system. Two assumptions will be considered: constant shadowing during the period of study and lognomal random shadowing.

This paper is organized as follows: the next section is dedicated to the system model description. In section III, two outage probability expressions are derived, the first one concerns the case of Rayleigh fading channels, the second expression is obtained when considering path loss, shadowing and fast fading. In section IV, simulation results are presented and discussed. Some conclusions are proposed in section V.

\section{System Model}

Consider a downlink, multicellular multiuser system. Each base station (BS) is equipped with M antennas and there are $\mathrm{K}$ users per cell with a single antenna each, as presented in Fig 1.

The zero forcing pre-equalization is a linear precoding technique that eliminates the multiuser interference at the transmitter level. It consists of multiplying the information vector by the $\mathrm{ZF}$ precoding matrix given by:

$$
\mathbf{W}=\mathbf{H}^{H}\left(\mathbf{H H}^{H}\right)^{-1},
$$




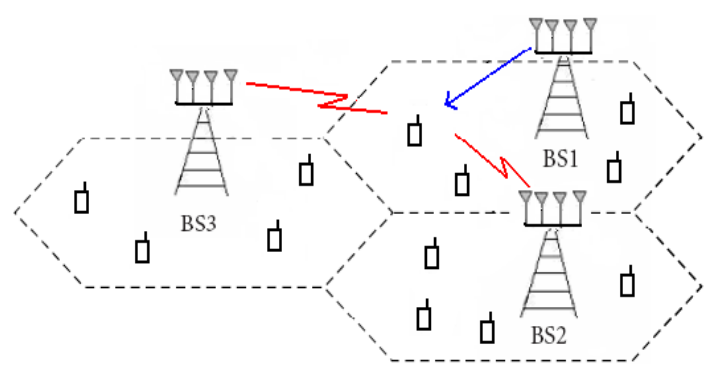

Fig. 1. System model

where $\mathbf{H}$ is the channel between the BS and the users of the cell and $H$ is the Hermitian operator. $\mathbf{H}$ is a $K \times M$ matrix with complex Gaussian distributed entries, i.e, $h_{i, j}$ the channel between the user $i$ and the antenna $j$ of the BS is $C N(0,1)$, the complex normal distribution. Hence the transmitted signal is given by:

$$
\mathbf{x}=\sqrt{P_{T}} \frac{\mathbf{W s}}{\sqrt{\delta}},
$$

where $P_{T}$ is the transmit power, $\mathbf{s}=\left[s_{1}, s_{2}, \ldots s_{K}\right]^{T}$ the symbol information vector intended to the $\mathrm{K}$ users and $\delta$ is a normalization factor introduced to satisfy the transmission power constraint and to ensure: $\|\mathbf{x}\|^{2}=P_{T}(\|$.$\| is the$ Euclidian norm). $\delta$ is given by:

$$
\delta=\|\mathbf{W} \mathbf{s}\|^{2} .
$$

The maximum number of users that can be served by a BS is $K=M$. When $K>M, M$ among the $K$ users can be scheduled to be served by the BS simultaneously. The scheduling algorithms are beyond the scope of this paper.

In a multicellular system ( $B \mathrm{BSs}$ ), the signal received by a user $k$ served by the BS 0 is given by:

$$
y_{k}=\sqrt{P_{0}} \mathbf{h}_{0, k} \frac{\mathbf{W}_{0} \mathbf{s}_{0}}{\sqrt{\delta_{0}}}+\sum_{j=1}^{B} \sqrt{P_{j}} \mathbf{h}_{j, k} \frac{\mathbf{W}_{j} \mathbf{s}_{j}}{\sqrt{\delta_{j}}}+n_{k},
$$

where $\mathbf{h}_{j, k}$ (a $1 \times M$ vactor) is the channel between the BS $j$ and user $k, \mathbf{W}_{j}$ is the precoding matrix used by the BS $j, \mathbf{s}_{j}$ is the information symbol vector intended to the users of BS $j, \delta_{j}$ is the normalization factor used by the BS $j$ and $n_{k}$ is the AWGN (Average White Gaussian Noise). $P_{j}$ is the power received from the BS $j$ including path-loss and shadowing terms and is given by:

$$
P_{j}=P_{T} A d_{j}^{-\eta} 10^{\frac{\xi_{j}}{10}},
$$

where $A$ is a constant, $d_{j}$ is the distance between the considered user and BS $j, \eta$ is the path-loss exponent and is characteristic of the propagation environment and $\xi_{j}$ is a Normal random variable with zero mean and standard deviation $\sigma$. From (4) we can write:

$$
y_{k}=\frac{\sqrt{P_{0}}}{\sqrt{\delta_{0}}} s_{0, k}+\sum_{j=1}^{B} \sqrt{P_{j}} \mathbf{h}_{j, k} \frac{\mathbf{W}_{j} \mathbf{s}_{j}}{\sqrt{\delta_{j}}}+n_{k} .
$$

The SINR at the user $k$ can, hence be written as:

$$
\gamma=\frac{P_{0}}{\delta_{0}\left(\sum_{j=1}^{B} P_{j} \frac{\left\|\mathbf{h}_{j, k} \mathbf{W}_{j}\right\|^{2}}{\delta_{j}}+\sigma_{n}^{2}\right)},
$$

where $\sigma_{n}$ is the noise variance.

In an interference limited system (e.g. in an urban environment) where the noise power can be neglected compared to the interference power, the SINR can be approximated as:

$$
\gamma \approx \frac{P_{0}}{\delta_{0}\left(\sum_{j=1}^{B} P_{j} \frac{\left\|\mathbf{h}_{j, k} \mathbf{W}_{j}\right\|^{2}}{\delta_{j}}\right)},
$$

\section{Outage Probability}

The outage probability is an important performance metric since it measures the probability of failure to ensure a required threshold for a given service. It can be defined as

$$
P_{\text {out }}=P\left[\gamma<\gamma_{t h}\right] \text {, }
$$

where $\gamma_{t h}$ is the SINR threshold value.

Writing the SINR given by (8) as:

$$
S I N R \approx \frac{X}{Y}
$$

where

$$
X=\frac{P_{0}}{\delta_{0}},
$$

and

$$
Y=\sum_{j=1}^{B} P_{j} \frac{\left\|\mathbf{h}_{j, k} \mathbf{W}_{j}\right\|^{2}}{\delta_{j}} .
$$

We need to calculate the probability density function (PDF) of $X$ and $Y$ to derive the outage probability expression.

\section{A. Constant shadowing assumption}

In this section, we will assume that the shadowing remains constant during the period of study. This assumption allows us to derive a simple closed form expression of the outage probability.

If we assume standard Gaussian information symbols, i.e the entries of $\mathbf{s}_{0}, s_{0, i}$ are $C N(0,1)$, the PDF of $X=\frac{P_{0}}{\delta_{0}}$ is a scaled F-distribution [7] that is given by:

$$
\begin{aligned}
f_{X}(x) & =\frac{P_{0}(M-K+1)}{K} F(x, 2(M-K+1), 2 K),(13) \\
& =\frac{M !}{(K-1) !(M-K) !} \frac{P_{0}^{K} x^{M-K}}{\left(P_{0}+x\right)^{M+1}} .
\end{aligned}
$$

In [3], it was shown that the $F$-distribution $F\left(x, n_{1}, n_{2}\right)$ can be approximated by a Chi-square distribution with degrees of freedom $n_{1}$. In our case $n_{1}=2(M-K+1)$. The PDF of $X$ can hence be expressed as:

$$
f_{X}(x) \approx \frac{K^{M-K+1}}{P_{0}^{M-K+1}(M-K) !} e^{-\frac{K x}{P_{0}}} x^{M-K} .
$$

To derive the density power function of the inter-cell interference power $Y$ given by (12), we can notice that $Y$ is the sum of positive random variables. Hence, using the central limit 
theorem for causal functions [5], the distribution of $Y$ can be approximated by a Gamma distribution that can be written as:

$$
f_{Y}(y)=\frac{y^{\nu-1} \exp \left(-\frac{y}{\mu}\right)}{\Gamma(\nu) \mu^{\nu}}
$$

where $\nu=\frac{\mathrm{E}[Y]^{2}}{\operatorname{var}(Y)}$ and $\mu=\frac{\operatorname{var}(Y)}{\mathrm{E}[Y]}$.

To derive the analytical expressions of the parameters $\nu$ and $\mu$, let us write $Y$ as:

$$
Y=\sum_{j=1}^{B} P_{j} z_{j}
$$

where

$$
z_{j}=\frac{\left\|\mathbf{h}_{j, k} \mathbf{W}_{j}\right\|^{2}}{\delta_{j}} .
$$

Assuming independent received signals from the interfering BSs, the mean and the variance of $Y$ can be written as:

$$
\mathrm{E}[Y]=\sum_{j=1}^{B} P_{j} \mathrm{E}\left[z_{j}\right] \quad, \quad \operatorname{var}(Y)=\sum_{j=1}^{B} P_{j}^{2} \operatorname{var}\left(z_{j}\right)
$$

To calculate the mean and the variance of $z_{j}$, we can write $z_{j}$ as:

$$
z_{j}=\frac{U}{V}
$$

where $U=\frac{1}{\delta_{j}}$ and $V=\frac{1}{\left\|\mathbf{h}_{j, k} \mathbf{W}_{j}\right\|^{2}}$. It can be seen that $U$ and $V$ have the same PDF as $\mathrm{X}$, hence, using the same Chi-square approximation as for the useful power PDF (15) (with $P_{0}=1$ in this case), $z_{j}$ can be seen as the ratio of two correlated Gamma distributed random variables $(U$ and $V$ ). From [4], and denoting $\alpha=M-K+1$, the mean and the variance of $z_{j}$ are given by:

$$
\begin{aligned}
\mathrm{E}\left[z_{j}\right] & =\frac{\left(1-\rho^{2}\right)+\alpha-1}{\alpha-1} \\
\operatorname{var}\left(z_{j}\right) & =\frac{(5 \alpha-4)\left(1-\rho^{2}\right)^{2}+2(\alpha-1)(\alpha-2)\left(1-\rho^{2}\right)}{(\alpha-1)^{2}(\alpha-2)}
\end{aligned}
$$

for $\alpha>2$, i.e, $M>K+1 . \rho=\frac{\operatorname{Cov}(U, V)}{\sqrt{\operatorname{var}(U) \operatorname{var}(V)}}=0.154$ is the correlation coefficient between the two correlated random variables $U$ and $V$. It can be obtained by simulations.

Defining $\theta=\left(1-\rho^{2}\right)$, the expressions (20) and (21) allows us to derive the parameters $\nu$ and $\mu$ that are given by:

$$
\begin{aligned}
\nu & =\left(1-\frac{2(2 \alpha-1) \theta^{2}-(\alpha-1)^{2}(\alpha-2)}{(5 \alpha-4) \theta^{2}+2(\alpha-1)(\alpha-2) \theta}\right) \frac{\left(\sum_{j=1}^{B} P_{j}\right)^{2}}{\sum_{j=1}^{B} P_{j}^{2}} \\
\mu & =\frac{(5 \alpha-4) \theta^{2}+2(\alpha-1)(\alpha-2) \theta}{(\alpha-1)(\alpha-2) \theta+(\alpha-1)^{2}(\alpha-2)} \frac{\sum_{j=1}^{B} P_{j}^{2}}{\sum_{j=1}^{B} P_{j}}
\end{aligned}
$$

The outage probability can then be calculated as follows:

$$
\begin{aligned}
P\left(\gamma<\gamma_{t h}\right) & =\int_{0}^{\infty} \int_{0}^{\gamma_{t h} y} f_{X}(x) d x f_{Y}(y) d y \\
& =\int_{0}^{\infty} \frac{1}{(\alpha-1) !} \gamma\left(\alpha, \frac{K \gamma_{t h} y}{P_{0}}\right) \\
& \times \frac{y^{\nu-1} \exp \left(-\frac{y}{\mu}\right)}{\Gamma(\nu) \mu^{\nu}} d y
\end{aligned}
$$

where $\gamma(.,$.$) is the lower incomplete Gamma function.$

The outage probability expression is given by [2] (eq. 6.455-2, P.663):

$$
\begin{aligned}
P_{\text {out }}\left(\gamma_{t h}\right) & =\frac{\Gamma(\nu+\alpha)}{\Gamma(\nu) \mu^{\nu} \alpha !} \frac{P_{0}^{\nu}\left(K \gamma_{t h}\right)^{\alpha}}{\left(\gamma_{t h} K+\frac{P_{0}}{\mu}\right)^{\nu+\alpha}} \\
& \times{ }_{2} F_{1}\left(1, \nu+\alpha ; \alpha+1 ; \frac{\gamma_{t h} K}{\gamma_{t h} K+\frac{P_{0}}{\mu}}\right)
\end{aligned}
$$

where ${ }_{2} F_{1}(., . ; . ;$.$) is the Gauss' hypergeometric function [2].$

\section{B. Lognormal shadowing assumption}

In this section we will consider the case of a randomly varying shadowing following a lognormal distribution. We will derive an easily computable expression for the outage probability.

In this case, the received power $P_{j}$ is a lognormal random variable with PDF given by:

$$
f_{P_{j}}(x)=\frac{1}{a x \sigma \sqrt{2 \pi}} \exp \left(-\frac{\left(\ln (x)-a m_{j}\right)^{2}}{2 a^{2} \sigma^{2}}\right),
$$

where $a=\frac{\ln 10}{10}$ and $m_{j}=\frac{1}{a} \ln \left(A P_{T} d_{j}^{-\eta}\right)$.

For the interference power distribution, the Gamma approximation is still valid, however new expressions of the parameters $\mu$ and $\nu$ will be given taking into consideration the lognormal variation of the received powers. Referring to the shadowing, we will call these parameters: $\mu_{s}$ and $\nu_{s}$.

Let us start by recalculating the mean and the variance of the interference power considering this new assumption.

The mean and variance of $\mathrm{Y}$ are given by:

$$
\mathrm{E}[Y]=\sum_{j=1}^{B} \mathrm{E}\left[P_{j}\right] \mathrm{E}\left[z_{j}\right]
$$

and

$$
\operatorname{var}(Y)=\sum_{j=1}^{B} \mathrm{E}\left[P_{j}^{2}\right] \mathrm{E}\left(z_{j}^{2}\right)-\mathrm{E}\left[P_{j}\right]^{2} \mathrm{E}\left[z_{j}\right]^{2} .
$$

From (26), we can derive $\mathrm{E}\left[P_{j}\right]$ and $\mathrm{E}\left[P_{j}^{2}\right]$ that are given by:

$$
\begin{aligned}
\mathrm{E}\left[P_{j}\right] & =e^{\frac{a^{2} \sigma^{2}}{2}} A P_{T} d_{j}^{-\eta}, \\
\mathrm{E}\left[P_{j}^{2}\right] & =e^{2 a^{2} \sigma^{2}}\left(A P_{T} d_{j}^{-\eta}\right)^{2} .
\end{aligned}
$$


Using the expressions (20) and (29), the mean of $Y$ can be derived and is given by:

$$
\mathrm{E}[Y]=e^{\frac{a^{2} \sigma^{2}}{2}} A P_{T} \frac{\theta+\alpha-1}{(\alpha-1)} \sum_{j=1}^{B} d_{j}^{-\eta} .
$$

From (20), (21) and (30), the variance of $Y$ can be calculated and is given by:

$$
\operatorname{var}(Y)=\left(A P_{T}\right)^{2} G\left(K, \sigma^{2}, \theta\right) \sum_{j=1}^{B} d_{j}^{-2 \eta},
$$

where

$$
\begin{aligned}
G\left(K, \sigma^{2}, \theta\right) & =e^{2 a^{2} \sigma^{2}}\left(\frac{6 \theta^{2}}{(\alpha-1)(\alpha-2)}+\frac{4 \theta}{\alpha-1}+1\right) \\
& -e^{a^{2} \sigma^{2}}\left(\frac{\theta^{2}}{(\alpha-1)^{2}}+\frac{2 \theta}{(\alpha-1)}+1\right) .
\end{aligned}
$$

Using the expressions of the mean (31) and the variance (32), we can easily derive the expressions of the parameters $\nu_{s}$ and $\mu_{s}$ that are given by:

$$
\begin{aligned}
& \nu_{s}=\frac{e^{a^{2} \sigma^{2}}(\theta+\alpha-1)^{2}}{(\alpha-1)^{2} G^{2}\left(K, \sigma^{2}, \theta\right)} \frac{\left(\sum_{j=1}^{B} d_{j}^{-\eta}\right)^{2}}{\sum_{j=1}^{B} d_{j}^{-2 \eta}} \\
& \mu_{s}=\frac{\left(A P_{T}\right)^{2} G\left(K, \sigma^{2}, \theta\right)(\alpha-1)}{e^{\frac{a^{2} \sigma^{2}}{2}}(\theta+\alpha-1)} \frac{\sum_{j=1}^{B} d_{j}^{-2 \eta}}{\sum_{j=1}^{B} d_{j}^{-\eta}} .
\end{aligned}
$$

From (25), the outage probability by considering the lognormal shadowing conditioned on the useful received power is given by:

$$
\begin{aligned}
P_{\text {out }}\left(\gamma_{t h} \mid P_{0}\right) & =C \frac{P_{0}^{\nu_{s}}}{\left(\gamma_{t h} K+\frac{P_{0}}{\mu_{s}}\right)_{s}+\alpha} \\
& \times{ }_{2} F_{1}\left(1, \nu_{s}+\alpha ; \alpha+1 ; \frac{\gamma_{t h} K}{\gamma_{t h} K+\frac{P_{0}}{\mu_{s}}}\right),
\end{aligned}
$$

where

$$
C=\frac{\Gamma\left(\nu_{s}+\alpha\right)}{\Gamma\left(\nu_{s}\right) \mu_{s}^{\nu_{s}} \alpha !}\left(K \gamma_{t h}\right)^{\alpha} .
$$

Averaging over the distribution of $P_{0}$, the outage probability can be written as:

$$
\begin{aligned}
P_{\text {out }}\left(\gamma_{t h}\right) & =C \int_{0}^{\infty} \frac{x^{\nu_{s}}}{\left(\gamma_{t h} K+\frac{x}{\mu_{s}}\right)^{\nu_{s}+\alpha}} \\
& \times{ }_{2} F_{1}\left(1, \nu_{s}+\alpha ; \alpha+1 ; \frac{\gamma_{t h} K}{\gamma_{t h} K+\frac{x}{\mu_{s}}}\right) \\
& \times \frac{1}{a x \sigma \sqrt{2 \pi}} \exp \left(-\frac{\left(\ln (x)-a m_{0}\right)^{2}}{2 a^{2} \sigma^{2}}\right) d x
\end{aligned}
$$

\section{SiMULATION RESULTS}

In this section, we will begin by verifying the sensitivity of the Gamma approximation given by (16) of the interference power PDF to the number of interfering BSs. Fig 2 shows a comparison between the Gamma distribution and the simulated PDF when considering 6 and 18 interfering BSs.
We can see that the approximation is all the more precise when the number of surrounding BSs is larger which is predictable since the Gamma approximation is based on the central limit theorem.

To emphasize the degradation of performance induced
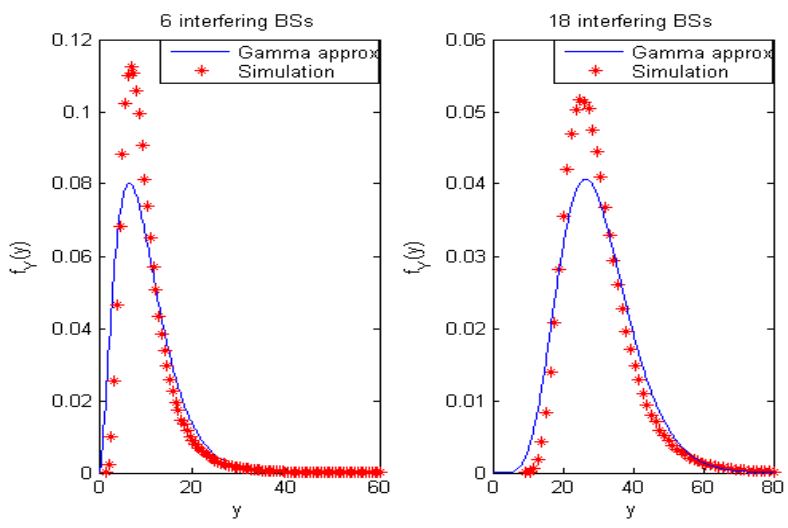

Fig. 2. Comparison between the simulated PDF and the Gamma approximation.

by the multicell interference, we simulated the outage probability of a single cell system compared to the multicell case (Fig. 3). For the multicell performance, the considered user receives a useful signal from its BS and an interfering signal from the two surrounding rings of BSs (18 interferers). All BSs transmit $P_{T}=20 \mathrm{~W}$, the cell radius is $R_{c}=1 \mathrm{Km}$ and the considered mobile station is at a distance $d=0.5 \mathrm{Km}$ from its serving BS. The standard deviation of the lognormal shadowing is chosen $\sigma=6 \mathrm{~dB}$ and the path-loss exponent is $\eta=3.41$. Each $\mathrm{BS}$ serves 4 single antenna users simultaneously. The total bandwidth allocated to the 4 users is $W=10 \mathrm{MHz}$. It can be seen that for an outage probability of $10 \%$ we have a loss of more than $10 \mathrm{~dB}$ in the SINR.

Fig. 4 presents a comparison between simulated and analytical

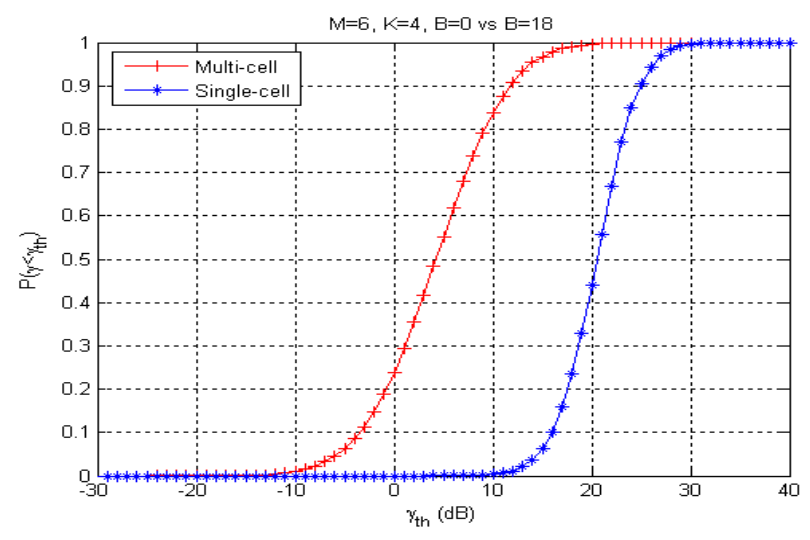

Fig. 3. Comparison between the ZF precoding performance in a single cell system versus multicell system.

performance of the ZF precoding without shadowing. As for the previous figure, we considered cellular system where the 
BSs are equipped with 6 antennas and we assumed 4 active single antenna terminals per cell. These values correspond to a typical urban environment. It can be seen that there is a good match between simulated and analytical results.

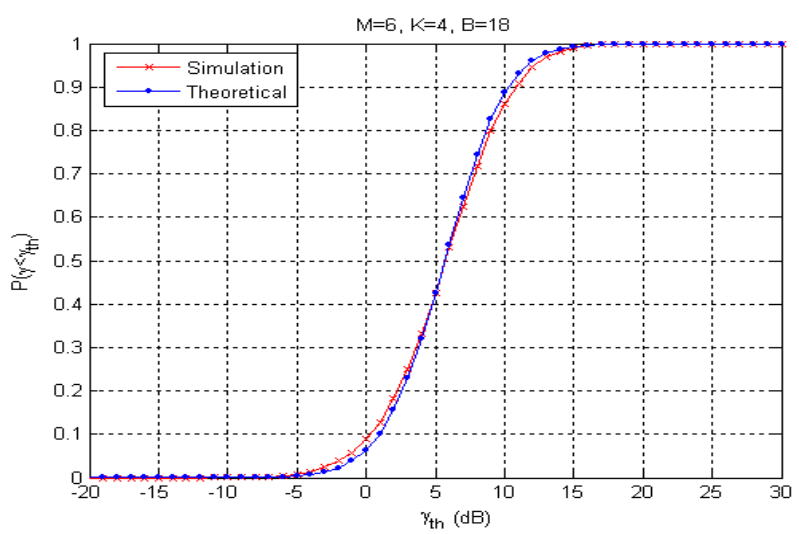

Fig. 4. $P_{\text {out }}$ versus SINR threshold for a multiuser multicellular ZF precoded system (constant shadowing).

Fig. 5 presents a comparison between the performance of the ZF transmission technique when considering lognormal shadowing and without shadowing. We considered the same simulation parameters as for Fig 4 except the standard deviation of the lognormal shadowing which is set to $\sigma=4 \mathrm{~dB}$. From the figure, we can see the degradation of performance induced by the shadowing effect. In fact, for $10 \%$ of outage probability, we have a loss of $4 \mathrm{~dB}$ in the SINR at the receiver level.

We can also see that there is a good agreement between simulation and analytical results.

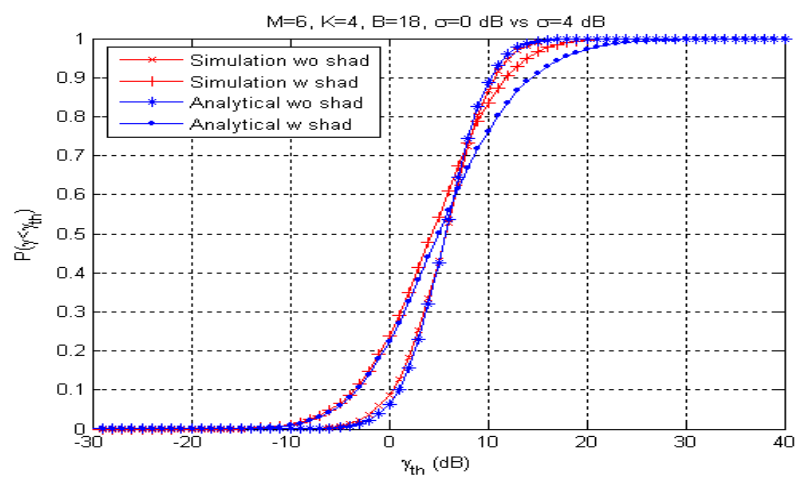

Fig. 5. Influence of the shadowing on the outage probability of the ZF precoded multiuser multicellular system.

\section{CONClusion}

In this paper, the performance of the zero forcing linear precoding technique was studied in terms of outage probability. Two expressions have been proposed. The first one corresponds to the case of a constant shadowing compared to the variation of the fast fading. The second expression takes into consideration the lognormal random variation of the shadowing along the time. Outage probability is an important parameter for the coverage analysis of cellular networks. As a future work, the newly derived expressions can be used for a dynamic study of our system that considers arrivals and departures of users in the cell. For this future analysis, the distribution function of the SINR is required in order to derive the average achievable throughput at a given distance.

\section{REFERENCES}

[1] M. Costa. Writing on dirty paper (corresp.). Information Theory, IEEE Transactions on, 29(3):439 - 441, May 1983.

[2] I. Gradshteyn and I.W.Ryzhik. Table of integrals series and products. Academic Press, Moscow, 1963.

[3] N. Johnson, S. Kotz, and N. Balakrishnan. Continuous univariate distributions. Wiley-Interscience, second edition, 1995.

[4] J. Panaretos, S. Psarakis, E. Xekalaki, and D. Karlis. The correlated gamma-ratio distribution in model evaluation and selection. MPRA Paper 6355, University Library of Munich, Germany, Sep. 2005.

[5] A. Papoulis. The Fourier Integral and Its Applications. McGraw-Hill, New York, NY, 1962.

[6] S. Ramanath, M. Debbah, E. Altman, and V. Kumar. Asymptotic analysis of precoded small cell networks. In INFOCOM, 2010 Proceedings IEEE, pages 1 -8, Mar. 2010.

[7] X. Shao, J. Yuan, and Y. Shao. Error performance analysis of linear zero forcing and mmse precoders for mimo broadcast channels. Communications, IET, 1(5):1067 -1074, Oct. 2007.

[8] E. Telatar. Capacity of multi-antenna gaussian channels. European Transactions on Telecommunications, 10(6):585-595, 1999.

[9] J. Wang, Z. Liu, Y. Wang, and X. You. Performance of the zero forcing precoding mimo broadcast systems with channel estimation errors. Journal of Electronics (China), 24:490-495, 2007. 10.1007/s11767-006-0070-0.

[10] H. Weingarten, Y. Steinberg, and S. Shamai. The capacity region of the gaussian mimo broadcast channel. In Information Theory, 2004. ISIT 2004. Proceedings. International Symposium on, page 174, June 2004.

[11] Y. Xu and T. Le-Ngoc. Optimal power allocation with channel inversion regularization-based precoding for mimo broadcast channels. EURASIP J. Adv. Signal Process, 2008:198:1-198:8, Jan. 2008.

[12] T. Yoo and A. Goldsmith. On the optimality of multiantenna broadcast scheduling using zero-forcing beamforming. IEEE Journal on Selected Areas in Communications, 24(3):528-541, Mar. 2006.

[13] L. Zheng and D. N. C. Tse. Diversity and multiplexing: A fundamental tradeoff in multiple-antenna channels. IEEE Trans. on Inform. Theory, 49(5):1073-1096, May 2003. 